\title{
ISCEV standard for clinical visual evoked potentials (2009 update)
}

\author{
J. Vernon Odom • Michael Bach • Mitchell Brigell • \\ Graham E. Holder • Daphne L. McCulloch • \\ Alma Patrizia Tormene $\cdot$ Vaegan
}

Received: 31 August 2009/Accepted: 2 September 2009/Published online: 14 October 2009

(C) Springer-Verlag 2009

\begin{abstract}
Visual evoked potentials (VEPs) can provide important diagnostic information regarding the functional integrity of the visual system. This document updates the ISCEV standard for clinical VEP testing and supersedes the 2004 standard. The major change in this revision is that test parameters have been made more precise to achieve better consistency of results within and between test centers. The ISCEV standard VEP protocols are defined for a single recording channel with a midline occipital active electrode. These protocols are intended for assessment

This document was prepared for the International Society for Clinical Electrophysiology of Vision (ISCEV) and document was approved by the membership of ISCEV in Abano Terme, Italy on July 10, 2009.
\end{abstract}

In memory of Vaegan (1943-2009): friend, dedicated ISCEV member, and member of this VEP standardization committee.

J. V. Odom $(\bowtie)$

West Virginia University Eye Institute, P.O. Box 9193,

Morgantown, WV 26506-9193, USA

e-mail: jodom@wvu.edu

M. Bach

Universitäts-Augenklinik, University of Freiburg,

Freiburg, Germany

M. Brigell

Novartis Institutes for BioMedical Research, Inc.,

Cambridge, MA, USA

G. E. Holder

Moorfields Eye Hospital, London, UK of prechiasmal function; additional electrode sites are recommended for evaluation of chiasmal and postchiasmal function. ISCEV has selected a subset of stimulus and recording conditions that provide core clinical information and can be performed by most clinical electrophysiology laboratories throughout the world. These are: 1 . Pattern-reversal VEPs elicited by checkerboard stimuli with large $1^{\circ}$ (i.e., 60 min of arc; min) and small $0.25^{\circ}$ (15 min) checks. 2. Pattern onset/ offset VEPs elicited by checkerboard stimuli with large $1^{\circ}$ (60 min) and small $0.25^{\circ}(15 \mathrm{~min})$ checks. 3. Flash VEP elicited by a brief luminance increment, a flash, which subtends a visual field of at least $20^{\circ}$.

Keywords Visual evoked potential .

Standard · Pattern reversal · Flash .

Pattern onset/offset - Visually evoked potential .

Visually evoked cortical potential

D. L. McCulloch

Vision Sciences, Glasgow Caledonian University,

Glasgow, Scotland, UK

\author{
A. P. Tormene \\ Department of Neurosciences, Ophthalmic Clinic, Padova \\ University, Padova, Italy \\ Vaegan \\ Visiontest Australia, Sydney, Australia
}




\section{Introduction}

VEPs (visual evoked potentials) are visually evoked electrophysiological signals extracted from the electroencephalographic activity in the visual cortex recorded from the overlying scalp. As visual cortex is activated primarily by the central visual field, VEPs depend on functional integrity of central vision at any level of the visual pathway including the eye, retina, the optic nerve, optic radiations, and occipital cortex.

This document updates the ISCEV standard for clinical VEP testing and supersedes the 2004 VEP standard [1]. The major change in the current standard compared with the previous VEP standard is that test parameters have been made more precise to achieve more consistency of results within and between test centers. Clinical VEP laboratories are encouraged to use the current standard method where possible. Reports of VEP recordings performed to the standard method given here should cite this 2009 standard. Where a method is used which deviates from the standard method, the deviations should be stated, together with any normative or reference data. Where the method used conforms to a previous ISCEV VEP standard, this may be cited instead.

The waveform of a VEP depends upon the temporal frequency of the stimulus. At rapid rates of stimulation, the waveform becomes approximately sinusoidal and is termed "steady state". At low temporal frequencies, the waveform consists of a number of discrete deflections and is termed a transient VEP. All ISCEV standard VEPs are transient.

This standard presents minimum protocols for basic clinical VEP recording. Three standard stimulus protocols are defined. The ISCEV standard VEP protocols are defined for a single recording channel with a midline occipital active electrode. If chiasmal or retrochiasmal disease is suspected, a three-channel montage, using the midline and two lateral active electrodes, is recommended in addition to the basic standard tests. Following a principle established in earlier standards [2-6], ISCEV has selected a subset of stimulus and recording conditions, which provide core clinical information that can be performed by most clinical electrophysiology laboratories throughout the world. These are
1. Pattern-reversal VEPs elicited by checkerboard stimuli with large $1^{\circ}$ (60 min of arc) and small $0.25^{\circ}$ (15 min) checks.

2. Pattern onset/offset VEPs elicited by checkerboard stimuli with large $1^{\circ}(60 \mathrm{~min})$ and small $0.25^{\circ}$ (15 min) checks.

3. Flash VEP elicited by a brief luminance increment, a flash, which subtends a visual field of at least $20^{\circ}$.

Pattern reversal is the preferred stimulus for most clinical purposes. Pattern-reversal VEPs are less variable in waveform and timing than the VEPs elicited by other stimuli. The pattern onset/offset stimulus is best suited for the detection of malingering and for use in patients with nystagmus. Flash VEPs are useful when poor optics, poor cooperation or poor vision makes the use of pattern stimulation inappropriate. To comply with this standard, at least one standard protocol should be included in every clinical VEP recording session so that all laboratories will have a common core of information that can be shared or compared.

ISCEV recognizes that VEPs may be elicited by a wide range of stimulus protocols that are not covered in the standard. Some of the widely used specialized VEPs and extended VEP protocols are listed in Table 1. Manufacturers are encouraged to produce equipment that can perform as many of these specialized tests as possible.

By limiting this standard to three protocols, the intention is that standard VEPs will be incorporated universally into clinical VEP testing along with additional tests and extended protocols that a laboratory may chose to use (Table 1). The standard does not require that all three protocols should be used for every investigation on every patient. In many circumstances,

Table 1 Specialized and extended VEP protocols not covered by the ISCEV Standard

Steady state VEP

Sweep VEP

Motion VEP

Chromatic (color) VEP

Binocular (dichoptic) VEP

Stereo-elicited VEP

Multi-channel VEP

Hemi-field VEP

Multifocal VEP

Multi-frequency VEP

LED Goggle VEP 
a single stimulus protocol will be appropriate. ISCEV actively encourages the use of additional protocols for clinical research, which may demonstrate that other tests are of equal or greater usefulness. This standard will be reviewed periodically and revised as needed.

The organization of this report is as follows:

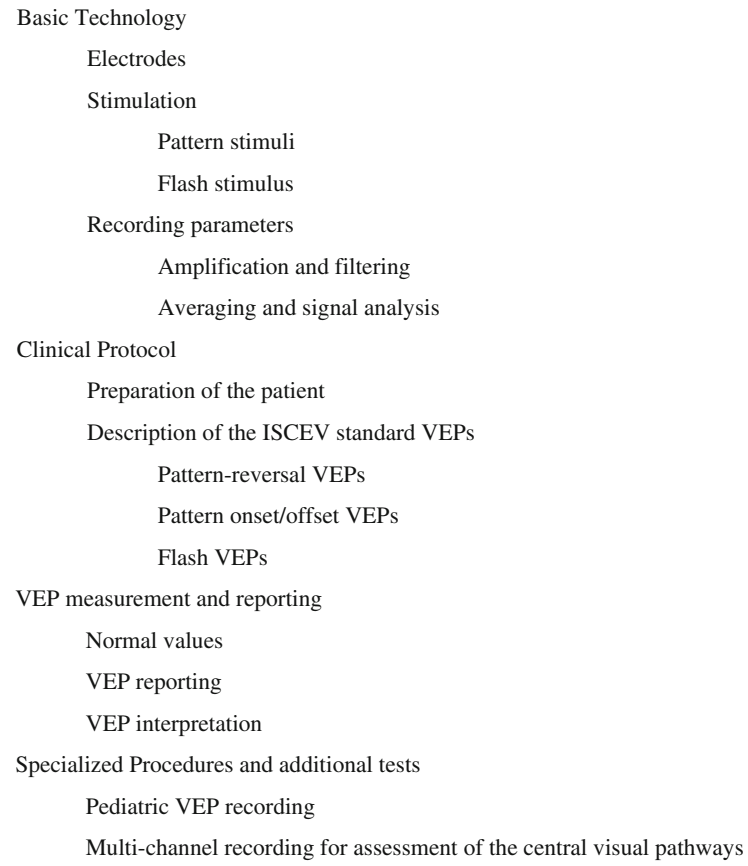

\section{Basic technology}

Electrodes

Skin electrodes such as sintered silver-silver chloride, standard silver-silver chloride, or gold disc electrodes are recommended for recording VEPs. The skin should be prepared by cleaning and a suitable paste or gel used to ensure good, stable electrical connection. The electrode impedances should be below $5 \mathrm{k} \Omega$ measured between 10 and $100 \mathrm{~Hz}$ and, to reduce electrical interference, they should not differ by more than $20 \%$ between electrode sites.

\section{Electrode placement}

The scalp electrodes should be placed relative to bony landmarks, in proportion to the size of the head, according to the International 10/20 system [7] (See Fig. 1a). The anterior/posterior midline measurements are based on the distance between the nasion and the inion over the vertex. The active electrode is placed on the scalp over the visual cortex at $\mathrm{Oz}$ with the reference electrode at Fz. A separate electrode should be attached to a relatively indifferent point and connected to the ground; commonly used ground electrode positions include the forehead, vertex $(\mathrm{Cz})$, mastoid, earlobe (A1 or A2), or linked earlobes.

Stimulus parameters

There are two major classes of standard VEP stimulation, flash, and pattern. The reader may refer to the ISCEV Calibration Guidelines [5] for guidance regarding the measurement and definition of stimulus parameters. Standard stimulus and recording conditions are described in the following sections and are summarized in Table 2A, B. All stimulus parameters should be calibrated either locally or by the manufacturer and regular recalibration is advised [5].

\section{Pattern stimuli}

The standard pattern stimulus is a high contrast black and white checkerboard. The viewing distance, typically between 50 and $150 \mathrm{~cm}$, can be adjusted to obtain a suitable field size and the required check sizes for any physical size of display screen.

\section{Field and check size}

Patterned stimuli are defined by a visual angle subtended by the side of a single check in degrees $\left(^{\circ}\right)$ or minutes of arc (min) subtended at the eye. One degree equals $60 \mathrm{~min}$ of arc. For standard pattern VEPs, two check element sizes should be used: $1^{\circ} \pm 20$ and $0.25^{\circ} \pm 20 \%$ per side. All checks should be square and there should be an equal number of light and dark checks. It is not necessary to use a square field but the aspect ratio between width and height should not exceed $4: 3$ and the field size should at least $15^{\circ}$ in its narrowest dimension. The stimulus field size should be specified in degrees of visual angle, with an indication of field shape (e.g., rectangular field $a^{\circ} \times b^{\circ}$, or a circular field of $c^{\circ}$ diameter or radius). A fixation point, when used, should be positioned at a corner of four checks which are located at the center of the field. 
Fig. 1 Electrode locations. a Location of active and reference electrodes for standard responses. The active electrode is located along the midline at $\mathrm{Oz}$. The reference electrode is located at location Fz. The subscript $z$ indicates a midline position. $\mathbf{b}$ The locations of the lateral active electrodes, $\mathrm{O}_{1}, \mathrm{O}_{2}$, $\mathrm{PO}_{7}$, and $\mathrm{PO}_{8}$ are indicated along with the midline active electrode location, $\mathrm{Oz}$

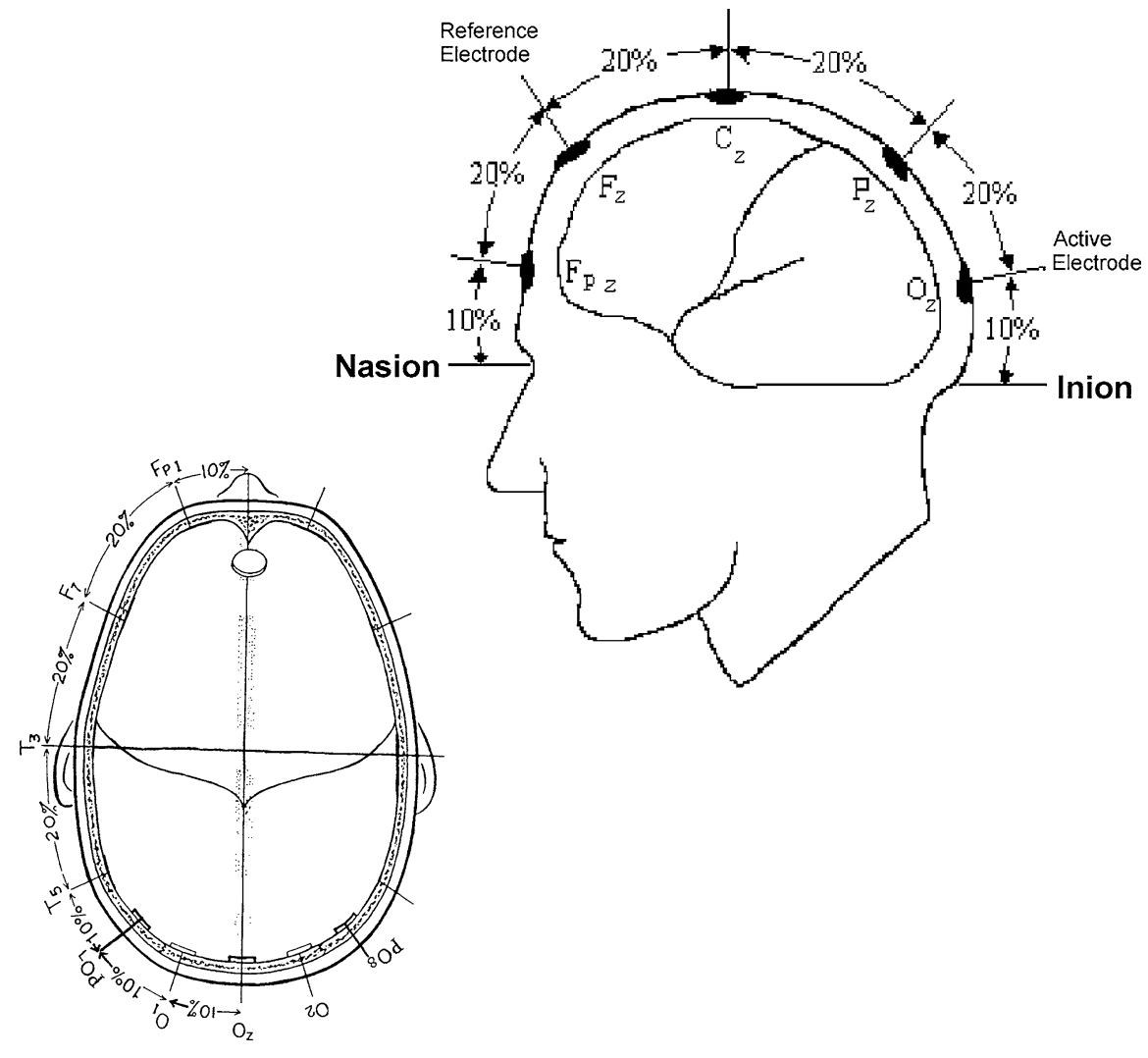

Luminance and contrast

The mean luminance of the checkerboard should be $50 \mathrm{~cd} \mathrm{~m}^{-2}\left(40-60 \mathrm{~cd} \mathrm{~m}^{-2}\right)$ and contrast between black and white squares should be high (as defined by Michelson contrast ${ }^{1}$ equal to or greater than $80 \%$ ). The luminance and contrast of the stimulus should be uniform between the center and the periphery of the field. However, we recognize that many optical and electronic systems do not provide truly uniform fields. Therefore, variation from center to periphery of up to $30 \%$ is acceptable. We encourage those following the standards to use stimulus displays that are as uniform as possible. The luminance of the background beyond the checkerboard stimulus field is not critical to the results. Lighting should be homogenous, with an average luminance approximately equal to the average stimulus luminance.

\footnotetext{
${ }^{1}$ Michelson contrast $=\{[\mathrm{Lmax}-\mathrm{Lmin}] /[\mathrm{Lmax}+\mathrm{Lmin}]\} \times$ $100 \%$, where $L$ denotes luminance, max denotes maximum of the white squares, and min denotes minimum of the black squares.
}

\section{Pattern-reversal stimuli}

For the pattern-reversal protocol, the black and white checks change phase abruptly (i.e., black to white and white to black) and repeatedly at a specified number of reversals per second. There must be no overall change in the luminance of the screen, which requires equal numbers of light and dark elements in the display, and no transient luminance change during pattern reversal. The large check $\left(1^{\circ}\right)$ and small check $\left(0.25^{\circ}\right)$ stimuli are specified by the check width (visual angle), the stimulus rate (in reversals per second), the number of reversals, the mean luminance, the pattern contrast, and the field size. A reversal rate of two reversals per second $( \pm 10 \%)$ should be used to elicit the standard pattern-reversal VEP. (Each full cycle consists of two reversals so this equates to a frequency of $1.0 \mathrm{~Hz}$.)

\section{Pattern onset/offset stimuli}

For pattern onset/offset, the checkerboard pattern is abruptly exchanged with a diffuse gray background. 
The mean luminance of the diffuse background and the checkerboard must be identical with no change of luminance during the transition from pattern to diffuse blank screen. This may be difficult to achieve. Pattern onset duration should be 200 ms separated by $400 \mathrm{~ms}$ of diffuse background. The ISCEV standard onset/offset response is the onset response. This temporal pattern ensures that the pattern onset response is not contaminated by the pattern offset response. The data acquisition system must indicate the appearance of the stimulus. At least two pattern element sizes should be used: checks of $1^{\circ}$ and $0.25^{\circ}$ per side.

\section{Flash stimulus}

The flash VEP should be elicited by a brief flash that subtends a visual field of at least $20^{\circ}$, presented in a dimly illuminated room. The strength (time-integrated luminance) of the flash stimulus should be 3 (2.7-3.3) photopic candelas seconds per meter squared $\left(\mathrm{cd} \mathrm{s} \mathrm{m}^{-2}\right)$. This can be achieved using a flashing screen, a hand held stroboscopic light or by positioning an integrating bowl (ganzfeld) such as that used for ERG tests [3] in front of the patient. The flash rate should be 1 per second $(1.0 \mathrm{~Hz} \pm 10 \%)$.

Recording parameters

\section{Amplification and filtering}

Amplification of the input signal by $20,000-50,000$ times is usually appropriate for recording the VEP. The input impedance of the preamplifiers must be at least $100 \mathrm{M} \Omega$ and the common mode rejection ratio should exceed $120 \mathrm{~dB}$. The amplifiers must be electrically isolated from the patient and must meet the current standards for safety for clinical biologic recording equipment in the user's country. The analogue signal should be digitized at a minimum sample rate of 500 samples per second per channel with a minimum resolution of 12 bits. Automatic artifact rejection based on signal amplitude should be used to exclude signals exceeding $\pm 50-100 \mu \mathrm{V}$ in amplitude. The amplifiers must return to baseline rapidly following artifactual signals.

Analogue high pass and low pass filters $[-3 \mathrm{~dB}$ points] should be set at $\leq 1 \mathrm{~Hz}$ (corresponding to a time constant $0.16 \mathrm{~s}$ or more) and at $\geq 100 \mathrm{~Hz}$, 
respectively. Analogue filter roll-off slopes should not exceed $12 \mathrm{~dB}$ per octave for low frequencies and $24 \mathrm{~dB}$ per octave for the high frequencies. While other filter settings may be required in particular circumstances, it must be realized that all analogue filters produce an apparent change in the timing or peak time of the components of the VEP particularly if low pass filters below $100 \mathrm{~Hz}$ are used. The use of notch or comb line frequency filters is strongly discouraged. Users should adhere to the current ISCEV Guidelines [5] that include details on the measurement of electrode impedance as well as amplifier filtering and gain.

\section{Averaging and signal analysis}

The number of sweeps per average depends upon the signal to noise ratio between the VEP and the background noise. In most clinical settings, the minimum number of sweeps per average should be 64. At least two averages should be performed to verify the reproducibility of each VEP. For infants and young children, a smaller number of sweeps per average may sometimes produce a clearer response. The longer recording time required to increase sample size introduces the possibility of increased variability due to loss of attention and/or increased movement.

\section{Analysis time}

The minimum analysis time (sweep duration) for all adult transient flash and pattern-reversal VEPs is $250 \mathrm{~ms}$ poststimulus. To analyze both the pattern onset and offset responses elicited by onset/offset stimuli, the analysis time (sweep duration) must be extended to $500 \mathrm{~ms}$. The VEP in infants has longer peak latencies and a longer sweep time will be required to adequately visualize the response.

\section{Clinical protocol}

Preparation of the patient

Pattern stimuli for VEPs should be presented when the pupils of the eyes are unaltered by mydriatic or miotic drugs. Pupils need not be dilated for the flash VEP. Extreme pupil sizes and any anisocoria should be noted for all tests. For pattern stimulation, the visual acuity of the patient should be recorded and the patient must be optimally refracted for the viewing distance of the screen. With standard electrodes and any additional electrode channels attached, the patient should view the center of the pattern field from the calibrated viewing distance.

Monocular stimulation is standard. This may not be practical in infants or other special populations, in such cases, binocular stimulation may be used to assess visual pathway function from both eyes. When a flash stimulus is used with monocular stimulation, care should be taken to ensure that no light enters the unstimulated eye. Usually, this requires a light-tight opaque patch to be placed over the unstimulated eye. Care must be taken to have the patient in a comfortable, well-supported position to minimize muscle and other artifacts.

\section{The ISCEV standard VEP waveforms}

VEP waveforms are age dependent. The description of standard responses reflects the typical waveforms of adults $18-60$ years of age. The time from stimulus onset to the maximum positive or negative deflection or excursion of the VEP will be referred to as the peak time. Historically, the term latency has been used to indicate the time from stimulus onset to the largest amplitude of a positive or negative deflection when referring to VEPs. In most areas of physiological recording and in electroretinography, the time from stimulus onset to the peak of a deflection has been referred to as the implicit time and latency referred to the time from stimulus onset to the beginning of a response. Recent ISCEV standards have tended to replace implicit time with peak time or peak latency $[1,4]$, because the meaning of the terms is more immediately apparent. We recognize that other terms have been used for this same concept.

\section{Pattern-reversal VEPs}

The pattern-reversal VEP waveform consists of N75, $\mathrm{P} 100$, and N135 peaks. These peaks are designated as negative and positive followed by the typical mean peak time (see Fig. 2). It is recommended to measure the amplitude of P100 from the preceding N75 peak. The P100 is usually a prominent peak that shows relatively little variation between subjects, minimal 


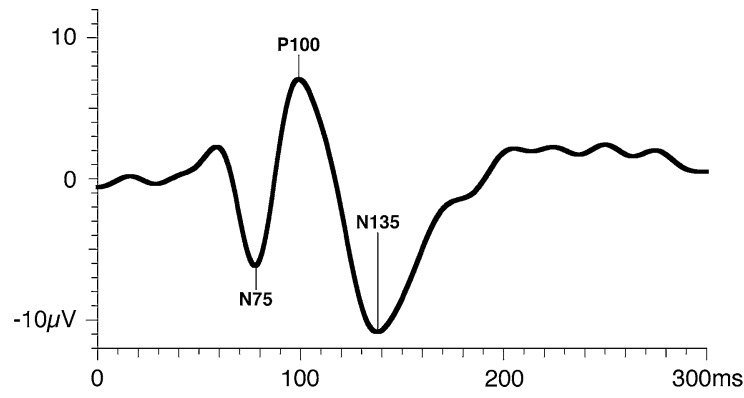

Fig. 2 A normal pattern-reversal VEP

within-subject interocular difference, and minimal variation with repeated measurements over time. P100 peak time is affected by nonpathophysiologic parameters such as pattern size, pattern contrast, mean luminance, signal filtering, patient age, refractive error, poor fixation, and miosis.

\section{Pattern onset/offset VEPs}

Pattern onset/offset VEPs show greater inter-subject variability than pattern-reversal VEPs. Pattern onset/ offset stimulation is effective for detection or confirmation of malingering and for evaluation of patients with nystagmus, as the technique is less sensitive to confounding factors such as poor fixation, eye movements or deliberate defocus. Standard VEPs to pattern onset/offset stimulation typically consists of three main peaks in adults; $\mathrm{C} 1$ (positive, approximately $75 \mathrm{~ms}$ ), $\mathrm{C} 2$ (negative, approximately $125 \mathrm{~ms}$ ), and C3 (positive, approximately $150 \mathrm{~ms}$ ) (see Fig. 3). Amplitudes are measured from the preceding peak.

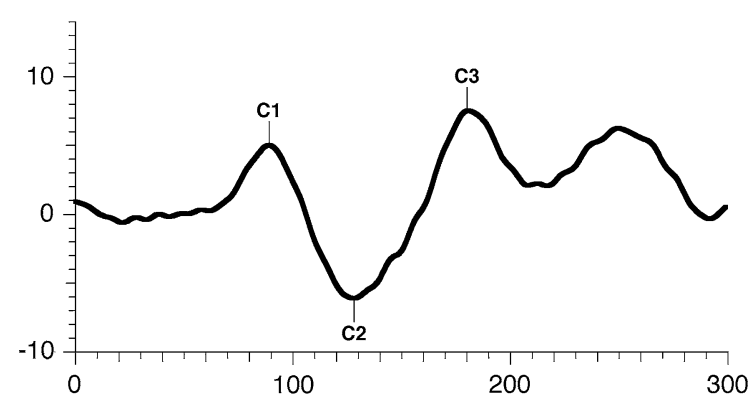

Fig. 3 A normal pattern onset/offset VEP. Note that with a $300 \mathrm{~ms}$ sweep only the pattern onset response is recorded
Flash VEPs

Flash VEPs are more variable than pattern VEPs across subjects, but are usually quite similar between eyes of an individual subject. They are useful for patients who are unable or unwilling to cooperate for pattern VEPs, and when optical factors such as media opacities prevent the valid use of pattern stimuli.

The VEP to flash stimulation consists of a series of negative and positive waves. The earliest detectable component has a peak time of approximately $30 \mathrm{~ms}$ poststimulus and components are recordable with peak latencies of up to $300 \mathrm{~ms}$. Peaks are designated as negative and positive in a numerical sequence (see Fig. 4). This nomenclature is recommended to differentiate the flash VEP from the pattern-reversal VEP. The most robust components of the flash VEP are the N2 and $\mathrm{P} 2$ peaks. Measurements of $\mathrm{P} 2$ amplitude should be made from the positive $\mathrm{P} 2$ peak at around $120 \mathrm{~ms}$ to the preceding $\mathrm{N} 2$ negative peak at around $90 \mathrm{~ms}$.

VEP measurement and reporting

\section{Normal values}

Although standardization should ensure similar VEP waveforms across laboratories, each laboratory must establish its own normative values using its own stimulus and recording parameters. The construction of a normal sample for laboratory norms should include the factors of age, sex, and interocular asymmetry. Adult normative data cannot be generalized to pediatric or elderly populations. Interocular comparison of amplitude and of peak time increases the sensitivity of the VEP to monocular conditions.

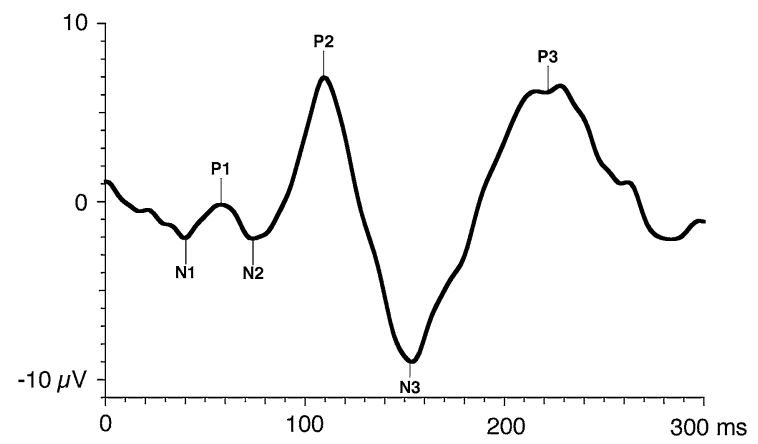

Fig. 4 A normal flash VEP 
Laboratory normal ranges should use descriptive statistics that do not assume a normal distribution, but are based on the calculation of the median and percentiles from the observed sample distribution. We recommend a $95 \%$ reference interval as the limit of normal (i.e., the range from 2.5 to $97.5 \%$ ).

\section{VEP reporting}

A minimum of two recordings of each VEP condition should be acquired, measured, and displayed to confirm reproducibility of the data. Reports using the standard VEP protocols should specify the following stimulus parameters; the field size of the stimulus, the strength (time-integrated luminance) of the flash or mean luminance of the pattern, the pattern element sizes and the contrast of pattern stimuli, the frequency of stimulation, and the eye tested. In addition, the following recording parameters should be reported; the filter settings and the locations of the positive (i.e., active) and negative (i.e., reference) and indifferent (i.e., ground) electrodes.

Traces should have a clear indication of polarity, time in milliseconds, and amplitude in microvolts. We recommend that VEP traces be presented as positive upwards. All VEP reports, including those for nonstandard responses (whether for local records or for publication), must report the peak time and amplitude measurements along with their normal values and the limits of normal. Reports should indicate whether the recordings meet this ISCEV standard.

\section{VEP interpretation}

VEP abnormalities are not specific and can occur in a wide variety of ophthalmological and neurological conditions. The interpretation should include statements about the normality and abnormality of the result in relation to normative data as well as comparison between the eyes or with previous records. The type of abnormality in the VEP should be described, and this should be related to the clinical picture and to other visual electrodiagnostic results.

Specialized procedures

\section{Pediatric VEP recording}

In principle, the stimulation and recording methods recommended in the ISCEV standard can be applied to all populations. However, in infants, young children, or people with disabilities, modifications to VEP recording methods and testing strategies may be required to optimize the quality and pertinence of the result to diagnosis, and visual assessment to the clinical question.

All VEPs in children should be compared with appropriate age-related normal values. When recording the VEP in young infants the sweep duration should be at least $500 \mathrm{~ms}$ poststimulus to record the full VEP waveform. By 6 months of age, the peak time of the main positive peak of the pattern-reversal VEP for large checks $\left(1^{\circ}\right)$ is usually within $10 \%$ of adult values.

Pediatric VEPs should be recorded when the infant or child is alert and attentive. Direct interaction with the child can help maintain attention and fixation, and two testers are beneficial; one to work with the child and the other to control data acquisition. Data quality and reliability will be improved if a recording trial can be paused or interrupted when fixation wanders and then resumed as the child resumes adequate fixation. To facilitate compliance, an infant may view the stimulus while held on a lap or over the shoulder. The order of stimulus presentation should be flexible and selected to ensure that responses most critical to the diagnostic question are obtained within an individual child's attention span. Binocular pattern stimulation, which facilitates attention and fixation, may be useful to evaluate overall visual function. Monocular testing to at least one stimulus is desirable to assess the function of each eye. It is particularly important to replicate VEPs in children to assure that the response measured is a reliable signal and not an artifact. Reports should note the degree of cooperation and arousal of the child. As for adults, additional channels of recording may be important for diagnosis of chiasmal and postchiasmal dysfunction. When pattern VEPs cannot be reliably recorded, flash testing, which is less dependent upon fixation, can usually be achieved.

\section{Multi-channel recording for assessment of the posterior visual pathways}

Multi-channel VEP recording is not required by the standard. However, intracranial visual pathway dysfunction may require multi-channel recording for accurate diagnosis. With dysfunction at, or posterior 
to, the optic chiasm, or in the presence of chiasmal misrouting (as seen in ocular albinism), there is an asymmetrical distribution of the VEP over the posterior scalp. Chiasmal dysfunction gives a "crossed" asymmetry whereby the lateral asymmetry obtained on stimulation of one eye is reversed when the other eye is stimulated. Retrochiasmal dysfunction gives an "uncrossed" asymmetry such that the VEPs obtained on stimulation of each eye show a similar asymmetrical distribution across the hemispheres. We suggest that pattern stimuli for multi-channel investigations of visual pathway dysfunction should be presented with a field of $30^{\circ}$ (double the minimum size required by this standard). A minimum of two channels is needed for detection of lateral asymmetries. We suggest a minimum of three active electrodes, two lateral electrodes placed at $\mathrm{O}_{1}$ and $\mathrm{O}_{2}$, and a third midline active electrode at $\mathrm{Oz}$. All three active electrodes should be referenced to Fz. Additional electrodes placed at $\mathrm{PO}_{7}$ and $\mathrm{PO}_{8}$, also referred to $\mathrm{Fz}$, may increase sensitivity to lateral asymmetries. The position of the lateral electrodes is illustrated in Fig. 1b. For all stimulus conditions, normative data should include amplitude and peak time comparisons between homologous left and right occipital channels. Particular caution is needed when interpreting multi-channel pattern-reversal VEPs because of paradoxical lateralization. This phenomenon, in which the response recorded at a lateral scalp location is generated by activity in the contralateral hemisphere of the brain, occurs with a large field, large check reversal stimulus and common reference recording to $\mathrm{Fz}$.

Acknowledgments ISCEV's standardization process requires the active participation of individual ISCEV members to act as consultants to the committee which writes the standard.

\section{References}

1. Odom JV, Bach M, Barber C, Brigell M, Holder G, Marmor MF, Tormene AP, Vaegan (2004) Visual evoked potentials standard. Doc Ophthalmol 108:115-123

2. Brown M, Marmor MF, Vaegan, Zrenner E, Brigell M, Bach M (2006) ISCEV standard for clinical electro-oculography (EOG) 2006. Doc Ophthalmol 113:205-212

3. Marmor MF, Fulton AB, Holder GE, Miyake Y, Brigell M, Bach M (2009) Standard for clinical electroretinography (2008 update). Doc Ophthalmol 118:69-77

4. Holder GE, Brigell MG, Hawlina M, Meigen T, Vaegan, Bach M (2007) ISCEV standard for clinical pattern electroretinography -2007 update. Doc Ophthalmol 2007(114): 111-116

5. Brigell M, Bach M, Barber C, Moskowitz A, Robson J (2003) Guidelines for calibration of stimulus and recording parameters used in clinical electrophysiology of vision. Doc Ophthalmol 107:185-193

6. Marmor MF, Hood DC, Keating D, Kondo M, Seeliger MW, Miyake Y (2003) Guidelines for basic multifocal electroretinography (mfERG). Doc Ophthalmol 106:105-115

7. American Clinical Neurophysiology Society (2006) Guideline 5: guidelines for standard electrode position nomenclature. J Clin Neurophysiol 23:107-110. Available at https:// www.acns.org/ 